\title{
Aquisição de medicamentos para a Doença de Alzheimer no Brasil: uma análise no sistema federal de compras, 2008 a 2013
}

\author{
The acquisition of medication to treat Alzheimer's disease in Brazil: \\ an analysis of federal purchases, 2008-2013
}

\author{
Roberta Dorneles Ferreira da Costa ${ }^{1}$ \\ Claudia Garcia Serpa Osorio-de-Castro ${ }^{2}$ \\ Rondineli Mendes da Silva ${ }^{2}$ \\ Aurélio de Araújo Maia ${ }^{3}$ \\ Mariana de Carvalho Barbosa Ramos ${ }^{3}$ \\ Rosângela Caetano ${ }^{1}$
}

${ }^{1}$ Departamento de Política, Planejamento e Administração em Saúde, Instituto de Medicina

Social, Universidade do Estado do Rio de Janeiro. R. São Francisco Xavier 524/ Pavilhão João Lyra Filho/7o/ D e E, Maracanã. 20550-013 Rio de Janeiro RJ Brasil. roberta_fdacosta@ yahoo.com.br ${ }^{2}$ Núcleo de Assistência Farmacêutica, Escola Nacional de Saúde Pública Sergio Arouca, Fiocruz. ${ }^{3}$ Departamento de Economia da Saúde, Secretaria Executiva, Ministério da Saúde.
Abstract The demographic transition in Brazil has led to substantial aging of the population and an increased prevalence of age-related diseases such as dementia and Alzheimer's Disease (AD). The Brazilian Ministry of Health finances AD medication and, since 2002, a Clinical Protocol and Therapeutic Guidelines (PCDT) for this condition have been made available. This study investigated the acquisition of medication for $A D$ in the Integrated System of Administration of General Services (SIASG) database. A profile of purchases, expenditures and prices from 2008 to 2013 was prepared. All medication and forms of treatment of $A D$ were investigated, including those not contained in the PCDT protocol. Unit prices were deflated to December 2013 by the IPCA (Brazilian Pricing Index). More than 47 million units of medication for $A D$ were acquired and expenditures attained 90.1 million Brazilian reals. The purchase of the various administration routes of rivastigmine were in the forefront. Medication not listed in the protocol represented $3 \%$ of expenditures and purchases resulting from health litigation were negligible. Over the period, a reduction of corrected weighted average prices of PCDT and non-PCDT medication was observed. Key words Alzheimer's disease, Expenses on medication, Drugs, Pharmaceutical services, Brazilian Unified Health System
Resumo O processo de transição demográfica brasileiro tem levado a substancial envelhecimento populacional e a aumento na prevalência de sindromes demenciais, entre as quais se destaca a Doença de Alzheimer (DA). Desde 2002, o tratamento farmacológico da doença possui Protocolo Clinico e Diretrizes Terapêuticas (PCDT) específico e os medicamentos recomendados são de financiamento pelo Ministério da Saúde. O estudo investigou a aquisição dos medicamentos utilizados no tratamento da DA a partir do Sistema Integrado de Administração de Serviços Gerais, traçando um perfil evolutivo das quantidades adquiridas, gastos e preços praticados no período de 2008 a 2013. Foram analisados os dados relativos a todas as apresentações adquiridas, inclusive as ausentes do PCDT. Os preços unitários foram deflacionados para dezembro/2013, pelo IPCA. Foram compradas mais de 47 milhões de unidades e gastos $R \$$ 90,1 milhões com medicamentos para DA. Destaca-se nos gastos a participação da rivastigmina em suas diversas apresentações. Medicamentos não indicados pelo PCDT representaram 3\% dos gastos, com percentuais de compra associados ao atendimento de ações judiciais pouco expressivos. No período, ocorreu redução dos preços médios ponderados corrigidos de todos os medicamentos, mesmo dos não presentes no PCDT.

Palavras-chave Doença de Alzheimer, Gastos com medicamentos, Assistência farmacêutica, SUS 


\section{Introdução}

No Brasil, o envelhecimento populacional vem ocorrendo de modo acelerado. Prevê-se que a população de maiores de 60 anos, correspondente a $10 \%$ do total em 2010 , atingirá 28,3 milhões (13,7\%) em 2020, e 52 milhões $(23,8 \%)$ de idosos em 2040, ou seja, quase um quarto do total de habitantes do país ${ }^{1}$.

Dentre as doenças relacionadas com o envelhecimento estão as demências. Metanálise recente $^{2}$ estimou que, na maioria das regiões do mundo, a prevalência das demências, padronizadas por idade, varia entre $5 \%$ e $7 \%$ nos maiores de 60 anos. Na América Latina, a prevalência é elevada, com cerca de 35,6 milhões de pessoas vivendo com demência em 2010, que deverão dobrar a cada 20 anos. Parte significativa desses quadros demenciais decorre de Doença de Alzheimer (DA), colocando esta condição como uma prioridade de saúde mundial.

No país, estudos apontam para prevalência de demência na população maior de 65 anos de $7,1 \%$, com a DA responsável por mais da metade dos casos ${ }^{3}$. A taxa de incidência varia entre os estudos e regiões brasileiras, de acordo com a estrutura etária das populações e os métodos usados em sua mensuração: de 7,7 por 1.000 pessoas-ano, em São Paulo ${ }^{4}$, a 14,8 por 1.000 pessoas-ano, no Rio Grande do Sul ${ }^{5}$. Considerando a prevalência de demência no Brasil e a população de idosos de aproximadamente 15 milhões de pessoas, em 2013, a estimativa era de 1,1 milhão nesse ano ${ }^{6}$.

A Doença de Alzheimer leva ao comprometimento das funções cognitivas, motoras e comportamentais, influenciando no dia a dia dos indivíduos e induzindo, em estágios avançados, a elevado grau de dependência ${ }^{7}$. A enfermidade tem impacto significativo sobre a família e cuidadores, por sua longa extensão e complexidade de manifestações funcionais, emocionais e consequências sociais ${ }^{4}$. A doença não tem cura e o manuseio da condição envolve o tratamento de sintomas cognitivos e comportamentais8.

Possui, também, importante impacto econômico para pacientes, familiares e para saúde pública, em decorrência dos custos indiretos, associados à perda ou redução dos ganhos do paciente e membros da família, e dos custos diretos relacionados com tratamento médico, serviços sociais, e necessidade de institucionalização ou de um cuidador formal, à medida que a doença atinge estágios avançados ${ }^{9}$. Segundo relatório mundial sobre o impacto econômico da $\mathrm{DA}^{10}$, os custos diretos totais da doença em todo mundo foram de U\$ 604 bilhões, em 2010. Apenas nos Estados Unidos, foram estimados em mais de U\$ 183 bilhões, com expectativa de aumento para US\$ 1,1 trilhões, em 2050. Parte desses custos relaciona-se ao tratamento farmacológico da doença, que no Brasil é coberto pelo Sistema Único de Saúde (SUS).

O Programa de Assistência aos Portadores da Doença de Alzheimer foi criado no âmbito do SUS, em 2002, devendo ser executado de forma integrada pelo Ministério da Saúde (MS) e Secretarias de Saúde dos Estados, Distrito Federal e Municípios, em cooperação com as redes estaduais e seus centros de referência em assistência à saúde do idoso ${ }^{11}$. O primeiro PCDT para o tratamento da DA foi instituído no mesmo ano, contendo os critérios diagnósticos da condição, medicamentos e doses a serem utilizadas ${ }^{12}$.

Em 2010, este PCDT foi atualizado e, no momento, vige a terceira versão, reatualizada em $2013^{6}$. Segundo o Protocolo, indica-se os inibidores de acetilcolinesterase donepezila, rivastigmina e galantamina, em diversas apresentações orais, para o tratamento da doença. Embora com propriedades farmacológicas levemente diferentes, estes fármacos inibem a degradação da molécula de acetilcolina, o neurotransmissor associado à função de memória, por bloquear a enzima acetilcolinesterase. Atuariam contribuindo para a melhoria cognitiva e comportamental, de modo a estabilizar as manifestações da doença, retardar sua progressão e a necessidade de institucionalização dos pacientes ${ }^{13,14}$. As evidências acumuladas apontam benefícios na demência leve à moderada, sem diferenças significativas de eficácia entre os três fármacos e com a substituição entre eles justificada pela intolerância ao medicamento ou contraindicações específicas ${ }^{15}$.

Existem ainda outros esquemas farmacológicos que incluem a rivastigmina na forma transdérmica e a memantina oral ${ }^{13}$. A ausência destes dois esquemas no PCDT tem determinado suas solicitações via demandas judiciais, agregando custos para o SUS em suas diversas instâncias governamentais.

Os medicamentos presentes no PCDT fazem parte do Componente Especializado da Assistência Farmacêutica (CEAF), cujo financiamento é feito pelo Ministério da Saúde. Ademais, em função de seu impacto econômico e importância sanitária, a partir de 2009, os inibidores da acetilcolinesterase definidos no PCDT passaram a ser objeto de Parcerias de Desenvolvimento Produtivo (PDP). Esta iniciativa objetiva a transferência 
de tecnologias de medicamentos considerados estratégicos para o sistema de saúde, principalmente daquelas em estágio maduro de seu ciclo de vida, como é o caso dos medicamentos para DA. De acordo com o MS, as PDP pretendem ainda racionalizar o poder de compra do Estado, mediante a centralização seletiva dos gastos na área da saúde, com vistas à sustentabilidade do SUS e à ampliação da produção no país de produtos estratégicos ${ }^{16}$.

Este estudo, de caráter exploratório, pretendeu investigar o conjunto de medicamentos para tratamento da DA adquiridos no Brasil e registrados no sistema federal de compras, traçando um perfil evolutivo das quantidades, gastos e preços praticados, no período de 2008 a 2013.

\section{Metodologia}

Os dados das compras foram obtidos dos registros do Sistema Integrado de Administração de Serviços Gerais (SIASG), de responsabilidade do Ministério do Planejamento, Orçamento e Gestão. Este Sistema possui informações referentes aos processos de compras realizados pelo governo federal, seus conveniados e aquelas instituições que utilizam o sistema do Comprasnet ${ }^{17}$. Exceto as aquisições de produtos da PDP e de laboratórios oficiais, que ocorrem via convênios específicos, todos os demais medicamentos adquiridos por qualquer órgão federal são registrados nesse Sistema. Medicamentos comprados pelas instâncias estaduais, financiados ou não pelo MS, só passaram a ter obrigatoriedade de registro no SIASG a partir de 2012.

O SIASG permite identificar os seguintes dados, além da especificação completa do item: data da compra; órgão responsável e sua localização geográfica; modalidade de aquisição (pregão ou dispensa de licitação) e justificativa para compra (uso regular ou atendimento de demanda judicial); quantidade de unidades compradas e preço unitário.

Foram extraídos dados relativos a todas as apresentações dos medicamentos com indicação para tratamento da DA, inclusive aquelas não presentes no PCDT, compreendendo: (1) donepezila: comprimido $5 \mathrm{mg}$ e $10 \mathrm{mg}$; (2) galantamina: cápsulas de liberação prolongada de $8 \mathrm{mg}, 16 \mathrm{mg}$ e $24 \mathrm{mg}$; (3) rivastigmina: cápsulas de $1,5 \mathrm{mg}$, 3mg, 4,5mg e $6 \mathrm{mg}$, solução oral de $2 \mathrm{mg} /$ $\mathrm{ml}$, adesivo transdérmico $4,6 \mathrm{mg} / \mathrm{dia} ; 9,5 \mathrm{mg} / \mathrm{dia}$; 13,3mg/dia, e 17,7mg/dia; e (4) memantina: comprimido de $10 \mathrm{mg}$.
O período analisado abrangeu de janeiro/2008 a dezembro/2013. Este intervalo de tempo cobre marcos importantes, em termos da Doença de Alzheimer. Em 2008, a galantamina passou a fazer parte do Componente de Medicamentos de Dispensação Excepcional (que se transformou posteriormente no Componente Especializado). Em 2009, foi assinada a primeira PDP relacionada aos medicamentos para tratamento desta doença. Por fim, houve atualizações do PDCT relacionado à DA, em 2010 e 2013.

Os preços unitários foram deflacionados para dezembro/2013, empregando-se a variação anual do Índice Nacional de Preços ao Consumidor Amplo (IPCA), calculado pelo IBGE e obtidos diretamente no Instituto de Pesquisa Econômica Aplicada (IPEA), disponível no site http://www. ipeadata.gov.br/IBGE, para permitir a comparabilidade, dada a extensão do período analisado e a importância da atualização monetária dos valores para avaliação de tendências. A opção pelo uso desse deflator tomou por base a Lei no. $10.742 / 2003$, que estabeleceu as normas de regulação do setor farmacêutico e definiu este índice para fins de ajuste nos preços dos medicamentos no país ${ }^{18}$.

As quantidades compradas, valores totais e preços médios ponderados dos medicamentos foram examinados segundo ano de aquisição, órgão responsável pela compra (Ministérios da Saúde, Educação e Defesa, e Secretarias estaduais e municipais, que utilizam do Comprasnet para melhorar as margens de negociação de preços), modalidade (pregão ou dispensa de licitação) e justificativa para compra (atendimento de demanda judicial). Foram elaboradas planilhas anuais, no aplicativo Microsoft Excel (Microsoft Corp., Estados Unidos), por medicamento/apresentação, permitindo a análise a partir de frequências absolutas e relativas no intervalo temporal examinado.

\section{Resultados}

No período 2008-2013, foram compradas mais de 47 milhões de unidades de medicamentos para tratamento da DA (Tabela 1), predominando as compras de donepezila $(77,4 \%)$. Houve tendência de aumento nas quantidades adquiridas de donepezila até 2012, com queda no último ano do período. As compras da galantamina também apresentam tendência de elevação constante, com as quantidades adquiridas em 2013 correspondendo a um aumento de mais de quinze 
vezes em relação a 2008. A rivastigmina, em todas as suas apresentações, incluindo aquelas não presentes no PCDT, apresenta uma tendência flutuante no período examinado, com redução significativa do volume comprado em 2012 e 2013, quando os produtos dos convênios de PDP passam a ser adquiridos. A memantina, mesmo não integrando o PCDT, sofreu aumento progressivo das unidades adquiridas (mais de 360 mil unidades entre 2008 e 2013), com crescimento de mais de cinco vezes no período.

Foram gastos mais de $\mathrm{R} \$ 90$ milhões com medicamentos para tratamento da doença no período (Tabela 2). Destaca-se, nos gastos, a participação da rivastigmina, em suas diversas apresentações: $46,4 \%$ do total. Cerca de 3\% dos

Tabela 1. Quantidades adquiridas segundo medicamentos utilizados no tratamento da Doença de Alzheimer, 2008-2013.

\begin{tabular}{|c|c|c|c|c|c|c|c|}
\hline Medicamentos & 2008 & 2009 & 2010 & 2011 & 2012 & 2013 & $\begin{array}{c}\text { Total } \\
\text { 2008-2013 }\end{array}$ \\
\hline donepezila & 10.656 & 776.184 & 344.488 & 12.643 .034 & 22.951 .316 & 38.540 & 36.764 .218 \\
\hline donepezila $5 \mathrm{mg}$ & 7.084 & 362.962 & 214.112 & 6.042 .638 & 9.092 .310 & 18.616 & 15.737 .722 \\
\hline donepezila $10 \mathrm{mg}$ & 3.572 & 413.222 & 130.376 & 6.600 .396 & 13.859 .006 & 19.924 & 21.026 .496 \\
\hline galantamina & 44.824 & 185.979 & 9.458 & 264.404 & 352.853 & 705.036 & 1.562 .554 \\
\hline galantamina $8 \mathrm{mg}$ & 10.940 & 48.655 & 6.166 & 78.524 & 138.189 & 383.044 & 665.518 \\
\hline galantamina $16 \mathrm{mg}$ & 16.278 & 70.800 & 1.848 & 113.648 & 51.632 & 169.696 & 423.902 \\
\hline galantamina $24 \mathrm{mg}$ & 17.606 & 66.524 & 1.444 & 72.232 & 163.032 & 152.296 & 473.134 \\
\hline rivastigmina* & 3.504 .083 & 528.306 & 1.679 .018 & 1.725 .692 & 753.207 & 639.070 & 8.829 .376 \\
\hline rivast $2 \mathrm{mg} / \mathrm{ml}$ & 6.056 & 2.476 & 3.654 & 560 & 2.074 & 4.660 & 19.480 \\
\hline rivast $1,5 \mathrm{mg}$ & 806.168 & 207.528 & 399.508 & 17.364 & 219.912 & 184.536 & 1.835 .016 \\
\hline rivast $3 \mathrm{mg}$ & 1.155 .031 & 153.824 & 418.328 & 992.316 & 177.924 & 191.760 & 3.089 .183 \\
\hline rivast $4,5 \mathrm{mg}$ & 759.054 & 68.100 & 452.550 & 15.488 & 227.851 & 89.326 & 1.612 .369 \\
\hline rivast $6 \mathrm{mg}$ & 775.974 & 62.888 & 354.508 & 641.532 & 88.756 & 91.308 & 2.014 .966 \\
\hline rivast $4,6 \mathrm{mg} / \mathrm{dia}^{* * \neq}$ & 900 & 8.850 & 11.430 & 24.640 & 7.590 & 21.160 & 74.570 \\
\hline rivast $9,5 \mathrm{mg} / \mathrm{dia}^{* *+}$ & 900 & 24.640 & 39.040 & 22.000 & 21.510 & 41.500 & 149.590 \\
\hline rivast $13,3 \mathrm{mg} / \mathrm{dia}^{* * \neq}$ & - & - & - & 7.992 & 7.590 & 14.820 & 30.402 \\
\hline rivast $17,7 \mathrm{mg} / \mathrm{dia}^{* * \neq}$ & - & - & - & 3.800 & - & - & 3.800 \\
\hline memantina $^{\ddagger}$ & 24.548 & 34.956 & 53.140 & 33.524 & 55.468 & 159.153 & 360.789 \\
\hline Total & 3.584 .111 & 1.525 .425 & 2.086 .104 & 14.666 .654 & 24.112 .844 & 1.541 .799 & 47.516 .937 \\
\hline
\end{tabular}

DA - Doença de Alzheimer; PCDT - Protocolo Clínico e Diretriz Terapêutica para DA; rivast. - rivastigmina

* Inclui todas as apresentações de cada medicamento, inclusive as ausentes do PCDT; ${ }^{* *}$ forma farmacêutica transdérmicas;

$\stackrel{*}{*}$ medicamento ausente do PCDT.

Tabela 2. Gastos com aquisições governamentais federais segundo medicamentos utilizados no tratamento da Doença de Alzheimer, 2008-2013 (em Reais deflacionados pelo IPCA de dezembro/2013).

\begin{tabular}{|c|c|c|c|c|c|c|c|}
\hline Medicamentos & 2008 & 2009 & 2010 & 2011 & 2012 & 2013 & $\begin{array}{c}\text { Total } \\
2008-2013\end{array}$ \\
\hline done & & 11.1 & 3.656 .18 & 00 & & 77.1 &, 16 \\
\hline galantamina & $625.187,70$ & $1.980 .407,21$ & $85.120,39$ & $2.345 .592,73$ & $2.743 .998,55$ & $5.034 .193,84$ & 12.814 \\
\hline rivastigmina* & $.229 .906,72$ & $3.260 .777,13$ & $9.523 .045,23$ & $8.376 .567,40$ & 2.865 .4 & 2.589 .2 & $41.844 .926,00$ \\
\hline $\begin{array}{l}\text { apresentações } \\
\text { indicadas pelo PCDT }\end{array}$ & $.208 .217,49$ & $2.960 .062,63$ & 9.082 .5 & $8.056 .641,82$ & 2.461 .3 & ,67 & $39.708 .206,55$ \\
\hline $\begin{array}{l}\text { apresentações na } \\
\text { forma transdérmica }\end{array}$ & $21.689,23$ & $300.714,49$ & $440.487,88$ & $358.514,58$ & $365.435,90$ & $649.877,60$ & $2.136 .719,45$ \\
\hline memantina & & & 102.05 & & & & $572.935,55$ \\
\hline Total & $16.085 .981,19$ & $16.453 .211,20$ & $13.366 .410,96$ & $23.044 .014,13$ & $13.308 .167,62$ & $7.851 .100,03$ & $90.108 .885,14$ \\
\hline
\end{tabular}

IPCA - Índice Nacional de Preços ao Consumidor Amplo; PCDT - Protocolo Clínico e Diretriz Terapêutica. * gastos totais, incluindo as apresentações ausentes do PCDT. 
Tabela 3. Compras federais de medicamentos utilizados no tratamento da Doença de Alzheimer e proporção de aquisições originadas por demanda judicial, Brasil, 2008-2013.

\begin{tabular}{|c|c|c|c|c|c|c|c|c|c|}
\hline \multirow[b]{2}{*}{ Medicamentos } & \multicolumn{3}{|c|}{2008} & \multicolumn{2}{|c|}{2009} & \multicolumn{2}{|c|}{2010} & \multicolumn{2}{|c|}{2011} \\
\hline & $\begin{array}{l}\text { No Total } \\
\text { Unidades }\end{array}$ & \multicolumn{2}{|c|}{$\begin{array}{c}\text { \% Demanda } \\
\text { Judicial }\end{array}$} & $\begin{array}{l}\text { No Total } \\
\text { Unidades }\end{array}$ & $\begin{array}{l}\text { \% Demanda } \\
\text { Judicial }\end{array}$ & $\begin{array}{l}\text { No Total } \\
\text { Unidades }\end{array}$ & $\begin{array}{c}\text { \% Demanda } \\
\text { Judicial }\end{array}$ & $\begin{array}{l}\text { No Total } \\
\text { Unidades }\end{array}$ & $\begin{array}{c}\text { \% Demanda } \\
\text { Judicial }\end{array}$ \\
\hline donepezila 5mg & 7.084 & \multicolumn{2}{|c|}{8,3} & 362.962 & 0,2 & 214.112 & 0,1 & 6.042 .638 & 0,0 \\
\hline donepezila 10mg & 3.572 & \multicolumn{2}{|c|}{10,5} & 413.222 & 0,0 & 130.376 & 0,0 & 6.600 .396 & 0,0 \\
\hline galantamina $8 \mathrm{mg}$ & 10.940 & \multicolumn{2}{|c|}{1,7} & 48.655 & 0,0 & 6.166 & 0,0 & 78.524 & 0,0 \\
\hline galantamina $16 \mathrm{mg}$ & 16.278 & \multicolumn{2}{|c|}{0,0} & 70.800 & 0,0 & 1.848 & 0,0 & 113.648 & 0,0 \\
\hline galantamina $24 \mathrm{mg}$ & 17.606 & \multicolumn{2}{|c|}{2,1} & 66.524 & 0,5 & 1.444 & 13,6 & 72.232 & 0,3 \\
\hline rivast. sol $2 \mathrm{mg} / \mathrm{mL}$ & 6.056 & \multicolumn{2}{|c|}{0,0} & 2.476 & 0,0 & 3.654 & 0,1 & 560 & 14,3 \\
\hline rivast. $1,5 \mathrm{mg}$ & 806.168 & \multicolumn{2}{|c|}{0,0} & 207.528 & 0,0 & 399.508 & 0,0 & 17.364 & 0,0 \\
\hline rivast. $3 \mathrm{mg}$ & 1.155 .031 & \multicolumn{2}{|c|}{0,0} & 153.824 & 0,0 & 418.328 & 0,0 & 992.316 & 0,0 \\
\hline rivast. $4,5 \mathrm{mg}$ & 759.054 & \multicolumn{2}{|c|}{0,0} & 68.100 & 0,0 & 452.550 & 0,0 & 15.488 & 0,0 \\
\hline rivast. $6 \mathrm{mg}$ & 775.974 & \multicolumn{2}{|c|}{0,1} & 62.888 & 0,6 & 354.508 & 0,2 & 641.532 & 0,2 \\
\hline Total PCDT & 3.557 .763 & \multicolumn{2}{|c|}{0,1} & 1.456 .979 & 0,2 & 1.982 .494 & 0,2 & 14.574 .698 & 0,0 \\
\hline memantina 10mg & 24.548 & \multicolumn{2}{|c|}{20,6} & 34.956 & 27,2 & 53.140 & 12,4 & 33.524 & 14,8 \\
\hline rivast. FT & 1.800 & \multicolumn{2}{|c|}{20,0} & 33.490 & 1,7 & 50.470 & 4,3 & 58.432 & 1,8 \\
\hline rivast. $4,6 \mathrm{mg} / \mathrm{dia}$ & 900 & \multicolumn{2}{|c|}{20,0} & 8.850 & 0,0 & 11.430 & 6,3 & 24.640 & 1,7 \\
\hline rivast. $9,5 \mathrm{mg} / \mathrm{dia}$ & 900 & \multicolumn{2}{|c|}{20,0} & 24.640 & 2,3 & 39.040 & 3,7 & 22.000 & 2,7 \\
\hline rivast. $13,3 \mathrm{mg} / \mathrm{dia}$ & 0 & \multicolumn{2}{|c|}{ - } & 0 & - & 0 & - & 7.992 & 0,0 \\
\hline rivast. $17,7 \mathrm{mg} / \mathrm{dia}$ & 0 & \multicolumn{2}{|c|}{ - } & 0 & - & 0 & - & 3.800 & 0,0 \\
\hline Total Fora PCDT & 28.148 & \multicolumn{2}{|c|}{19,2} & 101.936 & 9,9 & 154.080 & 5,7 & 150.388 & 4,0 \\
\hline & & & 12 & & & 2013 & & Total 2 & 2008-2013 \\
\hline Medicamentos & $\begin{array}{l}\text { No } \mathrm{T} \\
\text { Unid }\end{array}$ & $\begin{array}{l}\text { otal } \\
\text { ades }\end{array}$ & $\begin{array}{r}\% \mathrm{De} \\
\text { Jud }\end{array}$ & $\begin{array}{l}\text { manda } \\
\text { licial }\end{array}$ & $\begin{array}{l}\text { No Total } \\
\text { Unidades }\end{array}$ & $\begin{array}{r}\text { \% Dema } \\
\text { Judicia }\end{array}$ & & $\begin{array}{l}\text { No Total } \\
\text { Unidades }\end{array}$ & $\begin{array}{l}\text { \% Demanda } \\
\text { Judicial }\end{array}$ \\
\hline donepezila 5mg & 9.09 & 2.310 & & 0,0 & 18.616 & 0,0 & & 15.737 .722 & 0,01 \\
\hline donepezila 10mg & 13.85 & 9.006 & & 0,0 & 19.924 & 0,0 & & 21.026 .496 & 0,00 \\
\hline galantamina 8mg & & 8.189 & & 0,0 & 383.044 & 0,0 & & 665.518 & 0,03 \\
\hline galantamina $16 \mathrm{mg}$ & & 1.632 & & 0,0 & 169.696 & 0,0 & & 423.902 & 0,00 \\
\hline galantamina $24 \mathrm{mg}$ & & 3.032 & & 0,3 & 152.296 & 0,0 & & 473.134 & 0,34 \\
\hline rivast. sol $2 \mathrm{mg} / \mathrm{mL}$ & & 2.074 & & 0,0 & 4.660 & 87,6 & & 19.480 & 21,38 \\
\hline rivast. $1,5 \mathrm{mg}$ & & 9.912 & & 0,0 & 184.536 & 0,0 & & 1.835 .016 & 0,00 \\
\hline rivast. $3 \mathrm{mg}$ & & 7.924 & & 0,0 & 191.760 & 0,0 & & 3.089 .183 & 0,00 \\
\hline rivast. $4,5 \mathrm{mg}$ & & 7.851 & & 0,0 & 89.326 & 0,0 & & 1.612 .369 & 0,00 \\
\hline rivast. $6 \mathrm{mg}$ & & 8.756 & & 0,2 & 91.308 & 0,0 & & 2.014 .966 & 0,18 \\
\hline Total PCDT & 24.02 & 0.686 & & 0,0 & 1.305 .166 & 0,5 & & 46.897 .786 & 0,05 \\
\hline memantina $10 \mathrm{mg}$ & & 5.468 & & 14,7 & 159.153 & 3,5 & & 360.789 & 11,04 \\
\hline rivast. FT & & 6.690 & & 13,3 & 77.480 & 6,5 & & 258.362 & 5,42 \\
\hline rivast. $4,6 \mathrm{mg} / \mathrm{dia}$ & & 7.590 & & 20,6 & 21.160 & 0,6 & & 74.570 & 4,02 \\
\hline rivast. $9,5 \mathrm{mg} / \mathrm{dia}$ & & 1.510 & & 10,5 & 41.500 & 6,7 & & 149.590 & 5,21 \\
\hline rivast. $13,3 \mathrm{mg} / \mathrm{dia}$ & & 7.590 & & 13,8 & 148.20 & 14,6 & & 30.402 & 10,56 \\
\hline rivast. $17,7 \mathrm{mg} / \mathrm{dia}$ & & 0 & & - & 0 & - & & 3.800 & 0,00 \\
\hline Total Fora PCDT & & 8.848 & & 10,3 & 299.293 & 3,5 & & 877.513 & 6,17 \\
\hline
\end{tabular}

FT - formas transdérmicas; PCDT - Protocolo Clínico e Diretriz Terapêutica; rivast. - rivastigmina; rivast. sol. - rivastigmina solução oral. 
vastigmina solução oral. Os percentuais de compra associados ao atendimento de ações judiciais dos medicamentos ausentes do PCDT não são expressivos; apenas $11,0 \%$ das quantidades adquiridas de memantina e 5,4\% da rivastigmina em forma transdérmica possuem esta justificativa de compra. Embora os volumes de memantina adquiridas se elevem ao longo do período, a proporção de aquisições por demanda judicial foi se reduzindo progressivamente, atingindo a apenas $3,5 \%$ do total, em 2013. A risvastigmina na forma de adesivos transdérmicos apresenta tendência similar: crescimento das quantidades absolutas adquiridas (de 1.800 unidades em 2008, para mais de 77.000, em 2013), com redução nos gastos para atendimento de demanda judicial (de $20 \%$ para 6,5\%, no mesmo período).

Os preços médios unitários ponderados pelas quantidades e deflacionados para dezembro/2013 apresentaram queda no período, com proporções e em anos diversos (Tabela 4). No caso dos medicamentos sob PDP, apenas a donepezila mostrou redução importante do preço, diminuição que ocorreu já a partir do ano seguinte à sua introdução nesta estratégia, em 2010. A risvastigmina, ao contrário, apresentou aumento ou estabilização nos preços após assinatura da Parceria (2009). Dos medicamentos não recomendados pelo PCDT, apenas a memantina apresentou queda significativa nos preços unitários, em paralelo ao aumento no volume de compras.

A Tabela 5 examina o órgão responsável pelas compras de memantina e rivastigmina transdérmica. Não houve quaisquer aquisições pelo Ministério da Educação. O Ministério da Defesa foi sempre o principal comprador, independente do medicamento/apresentação, em proporção que atingiu a $91,4 \%$ do total adquirido, no caso da rivastigmina transdérmica de $9,5 \mathrm{mg} / \mathrm{dia}$. Ainda assim, nem sempre esses volumes de compra se expressaram na obtenção de um melhor preço médio. Nas apresentações transdérmicas, destaca-se também a participação dos entes infranacionais a partir de 2012, sobretudo no último ano examinado, com aquisições realizadas geralmente a preços médios inferiores aos dos ministérios. No caso da memantina, as SES/SMS apresentaram volume adquirido $(32,1 \%)$ apenas secundado pelo MD (53\%). Embora o MS tenha adquirido 53.792 unidades de memantina no período, quase $75 \%$ foram vinculadas a atendimento de demandas judiciais.

\section{Discussão}

Para a adequada apreciação dos resultados deste estudo, é importante ter em mente algumas altera-

Tabela 4. Preço médio ponderado pago nas compras federais de medicamentos para o tratamento da Doença de Alzheimer, segundo medicamento/apresentação e ano de aquisição, 2008-2013 (em Reais deflacionados pelo IPCA de dezembro/2013).

\begin{tabular}{|c|c|c|c|c|c|c|}
\hline Medicamentos/apresentação & 2008 & 2009 & 2010 & 2011 & 2012 & 2013 \\
\hline donepezila $5 \mathrm{mg}^{* *}$ & 15,87 & 13,57 & 12,65 & 0,96 & 0,32 & 1,79 \\
\hline donepezila $10 \mathrm{mg}^{* \ddagger}$ & 14,97 & 14,99 & 7,27 & 0,97 & 0,34 & 2,20 \\
\hline galantamina $8 \mathrm{mg}^{*}$ & 11,81 & 9,42 & 8,28 & 8,03 & 6,86 & 6,60 \\
\hline galantamina $16 \mathrm{mg}^{*}$ & 14,27 & 10,74 & 10,14 & 8,95 & 7,94 & 7,48 \\
\hline galantamina $24 \mathrm{mg}^{* \neq}$ & 14,97 & 11,45 & 10,62 & 9,65 & 8,5 & 8,12 \\
\hline rivastigmina $1,5 \mathrm{mg}^{*}$ & 3,25 & 3,74 & 4,05 & 4,02 & 2,62 & 2,25 \\
\hline rivastigmina $3 \mathrm{mg}^{*}$ & 3,76 & 4,57 & 4,66 & 4,45 & 3,46 & 2,77 \\
\hline rivastigmina $4,5 \mathrm{mg}^{*}$ & 4,30 & 5,35 & 5,30 & 5,19 & 3,68 & 3,07 \\
\hline rivastigmina $6 \mathrm{mg}^{* \neq}$ & 4,36 & 5,18 & 5,41 & 5,15 & 3,55 & 3,43 \\
\hline rivastigmina Sol $2 \mathrm{mg} / \mathrm{ml}^{*}$ & 263,26 & 319,06 & 328,92 & 329,80 & 56,10 & 86,82 \\
\hline rivastigmina patch $4,6 \mathrm{mg} / 24 \mathrm{~h}$ & 11,15 & 9,82 & 11,73 & 9,01 & 9,50 & 8,64 \\
\hline rivastigmina patch $9,5 \mathrm{mg} / 24 \mathrm{~h}$ & 12,95 & 11,06 & 11,20 & 10,54 & 12,09 & 11,12 \\
\hline rivastigmina patch $13,3 \mathrm{mg} / 24 \mathrm{~h}$ & - & - & - & 10,72 & 12,08 & 11,35 \\
\hline rivastigmina patch $17,4 \mathrm{mg} / 24 \mathrm{~h}$ & - & - & - & 10,15 & - & - \\
\hline memantina $10 \mathrm{mg}$ & 2,65 & 2,62 & 1,92 & 2,21 & 1,61 & 0,95 \\
\hline
\end{tabular}

IPCA - Índice Nacional de Preços ao Consumidor Amplo; PCDT - Protocolo Clínico e Diretriz Terapêutica; PDP - Parceria de Desenvolvimento Produtivo.

*apresentações presentes no PCDT; ₹ Apresentações objeto de PDP específica.

Nota: As marcações em negrito demarcam o ano em que se instituiu a PDP específica para o medicamento/apresentação. 
Tabela 5. Volume comprado e preço médio ponderado dos medicamentos/apresentações para o tratamento da Doença de Alzheimer fora do PDCT, segundo órgão comprador e ano de aquisição, 2008-2013 (em Reais deflacionados pelo IPCA de dezembro/2013).

\begin{tabular}{|c|c|c|c|c|c|c|c|}
\hline \multirow{2}{*}{ Órgão } & \multicolumn{2}{|c|}{ MS } & \multicolumn{2}{|c|}{ MD } & \multicolumn{2}{|c|}{ SES/SMS } & \multirow[b]{2}{*}{ Tota } \\
\hline & qtidade & PMP & qtidade & PMP & qtidade & PMP & \\
\hline \multicolumn{8}{|l|}{ memantina } \\
\hline 2008 & 19.002 & 2,64 & 5.546 & 2,66 & 0 & NA & 24.548 \\
\hline 2009 & 9.520 & 4,19 & 25.436 & 2,04 & 0 & NA & 34.956 \\
\hline 2010 & 6.586 & 2,27 & 26.562 & 2,19 & 19.992 & 1,42 & 53.140 \\
\hline 2011 & 4.952 & 2,77 & 26.412 & 2,18 & 2.160 & 1,41 & 33.524 \\
\hline 2012 & 8.172 & 2,18 & 11.896 & 1,69 & 35.400 & 1,45 & 55.468 \\
\hline 2013 & 5.560 & 1,27 & 95.393 & 0,89 & 58.200 & 1,00 & 159.153 \\
\hline Total & 53.792 & - & 191.245 & - & 115.752 & - & 360.789 \\
\hline \multicolumn{8}{|c|}{ risvastigmina FT 4,6 mg/dia } \\
\hline 2008 & 180 & 20,88 & 720 & 9,54 & 0 & NA & 900 \\
\hline 2009 & 8.850 & 10,24 & 0 & NA & 0 & NA & 8.850 \\
\hline 2010 & 720 & 9,72 & 10.710 & 12,61 & 0 & NA & 11.430 \\
\hline 2011 & 420 & 9,28 & 24.220 & 9,60 & 0 & NA & 24.640 \\
\hline 2012 & 1.560 & 8,99 & 4.950 & 10,54 & 1.080 & 9,36 & 7.590 \\
\hline 2013 & 120 & 8,34 & 16.840 & 8,72 & 4.200 & 8,34 & 21.160 \\
\hline Total & 11.850 & - & 57.440 & - & 5280 & - & 74.570 \\
\hline \multicolumn{8}{|c|}{ rivastigmina FT 9,5mg/dia } \\
\hline 2008 & 180 & 24,12 & 720 & 11,12 & 0 & NA & 900 \\
\hline 2009 & 570 & 10,93 & 24.070 & 11,55 & 0 & NA & 24.640 \\
\hline 2010 & 1.440 & 11,27 & 37.600 & 11,88 & 0 & NA & 39.040 \\
\hline 2011 & 600 & 10,92 & 21.400 & 11,24 & 0 & NA & 22.000 \\
\hline 2012 & 2.250 & 10,63 & 17.820 & 13,24 & 1.440 & 10,65 & 21.510 \\
\hline 2013 & 2.760 & 9,92 & 35.140 & 11,35 & 3.600 & 9,73 & 41.500 \\
\hline Total & 7.800 & - & 136.750 & - & 5040 & - & 149.590 \\
\hline \multicolumn{8}{|c|}{ rivastigmina FT $13,3 \mathrm{mg} / \mathrm{dia}$} \\
\hline 2011 & 0 & NA & 7.992 & 11,42 & 0 & NA & 7.992 \\
\hline 2012 & 1.050 & 10,65 & 5.940 & 13,37 & 600 & 10,74 & 7.590 \\
\hline 2013 & 2.160 & 9,75 & 11.460 & 11,82 & 1.200 & 9,72 & 14.820 \\
\hline Total & 3.210 & - & 25.392 & - & 1800 & - & 30.402 \\
\hline \multicolumn{8}{|c|}{ rivastigmina FT $17,7 \mathrm{mg} / \mathrm{dia}$} \\
\hline 2011 & 0 & NA & 3.800 & 10,82 & 0 & NA & 3.800 \\
\hline Total & 0 & $\mathrm{NA}$ & 3.800 & 10,82 & 0 & NA & 3.800 \\
\hline
\end{tabular}

FT - formas transdérmicas (patch); IPCA - Índice Nacional de Preços ao Consumidor Amplo; MD - Ministério da Defesa; MS Ministério da Saúde; SES/SMS- compras das Secretarias estaduais e municipais de saúde utilizando o SIASG ; NA - Não se aplica; qtidade - no de unidades adquiridas; PCDT - Protocolo Clínico e Diretriz Terapêutica; PMP - preço médio ponderado.

ções ocorridas ao longo do tempo no financiamento dos medicamentos utilizados no tratamento da DA. A partir da Portaria n 2.981 , os medicamentos do PCDT passaram a fazer parte do Componente Especializado da Assistência Farmacêutica, que incluiu a donepezila no grupo $1 \mathrm{~A}$, de aquisição centralizada pelo MS e fornecimento para as SES, enquanto galantamina e rivastigmina faziam parte do grupo $1 \mathrm{~B}$, de medicamentos financiados pelo MS mediante transferência de recursos financeiros às SES e Distrito Federal, que realizavam as aquisições necessárias ${ }^{19}$. Em 2012, como preparação para a aquisição via convênio dos produtos de PDP, a
Portaria $\mathrm{n}^{\circ} 1.103$ estabeleceu a aquisição centralizada da rivastigmina nas apresentações cápsula de $1,5 \mathrm{mg}$, 3mg, 4,5mg e $6 \mathrm{mg}^{20}$. Não foi surpresa constatar que, em 2013, a Portaria no 1.554 produziu nova alteração na posição da rivastigmina no elenco do CEAF, incluindo-a no grupo $1 \mathrm{~A}$, exceto a apresentação em solução oral que continuou fazendo parte do grupo $1 \mathrm{~B}^{21}$. Esse perfil de financiamento e as alterações ocorridas determinam que as quantidades e gastos associados às compras dos medicamentos presentes no PCDT representam apenas parte do que é financiado pelo SUS para o tratamento dos pacientes com DA. 
Observou-se progressivo crescimento das quantidades totais dos medicamentos comprados até 2012, que atinge inclusive os medicamentos para DA ausentes do PCDT. O grande volume de compras de donepezila em 2012 ( $81 \%$ a mais de unidades que em 2011), com formação de estoques, pode explicar a importante redução das quantidades compradas em 2013. As compras da galantamina presentes no SIASG correspondem apenas a uma parcela do total financiado pelo MS, mas mesmo assim quase triplicaram, só considerando o período de 2011 a 2013 (de 264.404 para 705.036 unidades). Os entes subnacionais podem se utilizar do sistema de compras federais para tentar obter melhores preços unitários de aquisição. Das 1.516.898 unidades de galantamina adquiridas no período, $85,2 \%$ corresponderam a aquisições feitas por esses entes, com destaque para a SES do Ceará.

A rivastigmina passou a ser objeto de PDP em 2009, com previsão de produção local a cargo do Instituto Vital Brazil. Já as PDP da donepezila e galantamina, assinadas, respectivamente, em 2010 e 2013, não redundaram ainda em produção local. Houve grande compra federal, de mais de 3,5 milhões de unidades de rivastigmina, em 2008, ano que antecedeu a PDP. A queda nas compras registradas no SIASG, em 2012 e 2013, explica-se pela aquisição de produtos da PDP desde 2012, que se realizam mediante convênios e não se encontram registradas neste Sistema. Foram adicionalmente compradas 26.566 .350 unidades anuais de rivastigmina, nas apresentações de 1,5mg; 3mg; 4,5mg e $6 \mathrm{mg}$. Ou seja, considerando estes quantitativos, não houve queda e sim aumento das unidades adquiridas: de 1.725.692, em 2011, para mais de 27 milhões em 2012 e 2013 22,23.

Os gastos apresentaram tendência similar às quantidades compradas, com relevância para a participação da rivastigmina (46,4\% do total). Se somarmos aos gastos do medicamento registrado no SIASG àqueles decorrentes da compra dos produtos dos convênios de PDP a partir de 2012, o gasto total com aquisições diretas de medicamentos para DA ascenderia a $\mathrm{R} \$ 156.069 .761,24$, com este fármaco representando $67,7 \%$ desse total.

Gastos com medicamentos representam um desafio para as políticas de saúde, principalmente quando se referem a condições crônicas cuja prevalência tende a aumentar com o envelhecimento da população ${ }^{24}$. Segundo Vieira e Mendes ${ }^{25}$, utilizando dados do MS, os gastos com aquisição de medicamentos, no período de 2002 a 2006, aumentaram em quase $124 \%$, enquanto os gastos totais em saúde se elevaram tão somente 9,6\%.
Carias et al. ${ }^{26}$ referem que os valores gastos com medicamentos do Componente Especializado, aos quais pertencem aqueles aqui estudados, representam, a cada ano, parcela maior no orçamento da saúde. Não raramente, esses medicamentos apresentam alto custo unitário, devido aos avanços técnico-científicos incorporados, ou elevado custo agregado, decorrente de seu uso frequente em condições crônicas, como é o caso da DA.

Conforme estabelecido no conceito do CEAF, os PCDT publicados pelo Ministério da Saúde são documentos que devem estabelecer as linhas de cuidado para cada uma das doenças pertencentes ao Componente ${ }^{19}$, devendo ser periodicamente revisados. Estes protocolos pretendem qualificar a atenção aos usuários do SUS e criar mecanismos para garantia de uma prescrição segura e eficaz, na medida em que se apoiem nas melhores evidências disponíveis sobre a eficácia, efetividade, segurança e qualidade do fármaco e considerem as necessidades, comodidade posológica, custo e disponibilidade no mercado ${ }^{27}$.

Diversas compras de medicamentos não conformes ao PCDT - rivastigmina em apresentações transdérmicas e memantina - têm ocorrido, apontando para a existência de um conjunto de pacientes sendo tratados fora do recomendado. Trabalho realizado no Rio Grande do Sul examinou a adesão dos padrões de prescrição ao protocolo nacional da DA. O exame de 1.399 prescrições de inibidores da colinesterase, realizadas entre janeiro de 2005 e outubro de 2007, por um comitê de especialistas regionais, mostrou que apenas 25\% delas estavam conforme as recomendações do PCDT, com o diagnóstico de outros tipos de demência (principalmente demências de origem vascular ou por Doença de Parkinson), DA grave e performance cognitiva não compatível com os padrões do PCDT, os principais motivos de não conformidade ${ }^{28}$.

Em contrapartida, é relevante lembrar que os adesivos cutâneos possuem maior facilidade de administração e permitem a redução de efeitos adversos gastrintestinais presentes nas formulações orais, características importantes considerando a faixa etária mais atingida pela $\mathrm{DA}^{13,14}$. Em diversos países, é a principal forma farmacêutica utilizada. Já a memantina está indicada na doença moderada à grave ${ }^{29}$, cujo tratamento não se encontra incluso no PCDT, o que pode indicar a necessidade de revisão deste protocolo, já que a indicação é coberta em diversos sistemas de saúde, incluindo o inglês e canadense ${ }^{13}$.

Os medicamentos envolvidos nas compras sem conformidade com o PCDT possuem preços 
comparativamente mais elevados, estão há menos tempo no mercado, e alguns ainda protegidos por patente, como é o caso dos adesivos cutâneos. Suas compras responderam por 3\% dos gastos totais e a menos de $0,2 \%$ das unidades adquiridas no período 2008-2013. Nessas condições, o MS não pode exercer seu poder de compra associado a aquisições centralizadas. Muitas vezes, também, são aquisições relacionadas ao atendimento de demandas judiciais, em pequenas quantidades e a preços mais elevados.

A judicialização dos medicamentos continua como fenômeno bastante presente no Brasil, influenciando diretamente na gestão da assistência farmacêutica, na medida em que impacta nos gastos, desloca prioridades e atinge princípios do SUS, como a equidade ${ }^{30}$. Nesse sentido, salientase o fato referido por Pepe et al. de que grande parte dos medicamentos solicitados judicialmente encontra-se nos elencos de listas oficiais, com destaque para aqueles do CEAF ${ }^{31}$.

Estudos sobre judicialização do acesso mostram que a presença nos protocolos do SUS aumenta a chance do produto ser comprado por vias normais da gestão, já que os PCDT auxiliam os gestores de saúde na aquisição e dispensação de medicamentos, cumprindo papel importante no gerenciamento da assistência farmacêutica, bem como nos aspectos legais envolvidos no acesso a medicamentos e na assistência como um todo ${ }^{27}$. Esperar-se-ia, portanto, que a compra dos medicamentos não conformes ao PCDT de DA estivesse associada ao atendimento de demandas judiciais, o que não se verificou. A maior proporção deles foi comprada de forma regular, por pregão eletrônico. Além disto, verificou-se certa tendência de queda nas compras associadas a essa justificativa, ao longo do período estudado.

Chama atenção o papel desempenhado pelo Ministério da Defesa nas compras de medicamentos ausentes do PCDT, sugerindo que este ente não atua em conformidade com as diretrizes nacionais recomendadas para o tratamento dos indivíduos com DA. Isso se torna ainda mais relevante porque nenhuma das aquisições deste ministério adveio de atendimento a ação judicial. Por outro lado, é importante ser lembrada a cobertura diferenciada que o MD possui em relação a seus assistidos, que se mantêm atendidos mesmo após a aposentadoria da vida militar ativa e que se estende a dependentes e pensionistas. A disponibilidade de informações presentes no SIASG não permite o aprofundamento dos determinantes das aquisições pelo $\mathrm{MD}$, mas reforça desde já a importância de que as diretrizes sejam elaboradas contando com ampla participação de todos os atores relevantes envolvidos e fortemente apoiadas em evidências científicas de elevada qualidade, bem como a necessidade de sua divulgação abrangente a profissionais, usuários e gestores dos diferentes níveis.

Os anticolinesterásicos, indicados no tratamento da DA, são produtos antigos no mercado, com patentes vencidas ou em fase de vencimento, o que tende a impactar nos seus preços. Nossos resultados mostram que praticamente todos os medicamentos estudados apresentaram queda dos preços médios unitários no período, ainda que com velocidades e em proporções bastante diversas.

A significativa queda nos preços da donepezila - de R\$ 12,65, em 2010, para valores inferiores a $\mathrm{R} \$ 2,00$, a partir de 2011, quando se completou o processo de centralização de compras pelo MS - possivelmente decorreu do grande aumento do volume comprado, permitindo melhores margens de negociação com os produtores. Especificamente no caso da rivastigmina, verifica-se redução mais acentuada a partir do momento em que se inicia a aquisição dos produtos oriundos dos convênios de PDP. Embora o impacto direto das compras oriundas da transferência tecnológica não se encontre diretamente representado nas aquisições registradas no SIASG, a perspectiva concorrencial decorrente de sua disponibilidade pode estar se refletindo no observado ${ }^{16}$, empurrando os preços para baixo. Mesmo não sendo a solução oral objeto de PDP, houve também redução importante de seu preço a partir de 2012, possivelmente pela possibilidade de substituição, pelos prescritores, dessa apresentação por outras produzidas via PDP.

Considerando-se a importância da realização de estudos que acompanhem os gastos com a aquisição de compras governamentais, deve ser destacado que o SIASG revelou-se fonte de informação relevante para a observação das tendências ao longo do tempo. Contudo, a partir do momento que as aquisições oriundas das PDP comecem a se fazer mais presentes, torna-se necessária a utilização de outras fontes de dados que reflitam o perfil de consumo e gastos que se originam dos convênios, cujo registro não se encontra disponível nessa base. Embora todas as compras sejam publicadas em Diário Oficial, seu conhecimento implica no monitoramento desta fonte, cujos dados não são tão facilmente acessíveis como aqueles associados a sistemas como o SIASG.

O SIASG é uma base federal de acesso público. A oportunidade de verificar a especificação 
dos itens e de conhecer o órgão comprador traz confiabilidade e rastreabilidade às informações, favorecendo de modo ímpar a transparência das compras públicas. Por outro lado, o estudo das aquisições de medicamentos no sistema federal de registro de compras parte do pressuposto de que todas as compras executadas por órgãos federais estejam obrigatoriamente presentes no SIASG, mormente aquelas realizadas pelo próprio Ministério da Saúde.

Em documento do MS sobre o CEAF publicado em $2014^{32}$, os gastos totais com donepezila atingiram valores de $\mathrm{R} \$ 54.627 .654,22$ para o ano de 2011. Isso contrasta fortemente com o registrado no SIASG e levantado neste estudo: $\mathrm{R} \$$ 12.209.021,00 (Tabela 2). No entanto, a análise dos gastos do CEAF mostra que esta considerável diferença de valores, de mais de $\mathrm{R} \$ 42$ milhões, é proveniente dos gastos deste medicamento registrados no Sistema de Informações Ambulatoriais (SIA-SUS). Valores deste medicamento foram lançados via Autorização de Procedimento de Alto Custo/Complexidade (APAC), a partir de 2010 até setembro de 2011, quando as primeiras distribuições provenientes da compra centralizada foram iniciadas. A donepezila (grupo 1A) corresponde a produto de aquisição por meio de processo centralizado no MS, com posterior distribuição aos estados. Uma possível demora nesse processo ocasionou o pagamento via APAC ou por ressarcimento de estoques ${ }^{33,34}$.

O complexo processo de financiamento do SUS, no qual ocorre migração de medicamentos para diferentes modalidades ao longo do tempo, traz dificuldades à rastreabilidade das informações e, ainda que inadvertidamente, compromete a transparência das compras públicas. Seria importante que o SIASG pudesse trazer elementos que ajudassem a verificação dos preços, volumes e gastos e dos mecanismos de compra ao longo do tempo.

Em relação às limitações do estudo, deve ser considerado que todo trabalho baseado em dados secundários é fortemente dependente do tipo e qualidade da informação presente nas bases, nem sempre suficiente para investigações adicionais. No caso do SIASG, os dados disponibilizados pelo Sistema são alimentados por uma diversidade de entes compradores, o que pode se refletir na completude e qualidade dos registros. Além disso, a estrutura do banco em questão no que se refere às variáveis presentes restringe por si só as possibilidades de aprofundamento de certas questões mencionadas no texto.

Destaca-se que as quantidades e valores apresentados no estudo não expressam os gastos totais federais com o financiamento da galantamina, em todo o período estudado, e com a rivastigmina, até 2012. Embora o financiamento desses fármacos seja federal, sua aquisição poderia ocorrer de forma descentralizada nos estados, demandando a necessidade de estudos ulteriores, que considerem a compra pelos entes infranacionais, para o adequado dimensionamento dos gastos públicos relacionados com o tratamento da DA no país.

\section{Considerações Finais}

Houve aumento relevante da quantidade de medicamentos adquiridos no período. Estima-se que a constante atualização do protocolo, definindo os critérios de indicação do tratamento farmacológico, e a centralização das compras pelo MS possam ter contribuído para uma possível expansão do tratamento da DA. Os gastos também se elevaram ao longo do tempo, o que demonstra o efeito da prevalência crescente dessa condição na população brasileira e, potencialmente, de um maior acesso ao tratamento.

Nota-se que os mecanismos de provisão do sistema (PDP) estão se multiplicando e o volume de compras dos medicamentos para DA aumentando. Por outro lado, a demanda judicial por esses medicamentos está diminuindo proporcionalmente, significando um direcionamento das compras de anticolinesterásicos no sentido da normalização dos mecanismos de aquisição. Até que ponto o aumento nos quantitativos comprados representa, por um lado, melhor acesso, ou por outro, sobreprescrição de medicamentos, não é possível revelar com base nos dados de compras. No entanto, seria importante investigar se os incrementos verificados estão atendendo às necessidades sanitárias do país.

Também levanta preocupações o volume de recursos que vem sendo consumido na aquisição de medicamentos para a DA fora do PCDT instituído pelo MS, sobretudo pelo Ministério da Defesa. Nesse sentido, o trabalho realizado não apenas aponta para um consumo fora do recomendado, mas sinaliza para potencial diferença de acesso entre a população assistida por diferentes entes de um mesmo e único governo. 


\section{Colaboradores}

RDF Costa, CGS Osorio-de-Castro e R Caetano participaram da concepção do estudo, análise dos dados, redação do artigo e aprovação da versão final. RM Silva participou da análise dos dados e revisão do texto final. AA Maia e MCB Ramos participaram do levantamento dos dados e revisão do manuscrito final.

\section{Referências}

1. Mendes ACD, Sá DA, Miranda GMD, Lyra TM, Tavares RAW. Assistência pública de saúde no contexto da transição demográfica brasileira: exigências atuais e futuras. Cad Saude Publica 2012; 28(5):955-964.

2. Prince $M$, Bryce R, Albanese E, Wimo A, Ribeiro $\mathrm{W}$, Ferri CP. The global prevalence of dementia: A systematic review and metaanalysis. Alzheimer's Dement 2013; 9(1):63-75.

3. Herrera Jr E, Caramelli P, Silveira ASB, Nitrini R. Epidemiologic survey of dementia in a community-dwelling Brazilian population. Alzheimer Dis Assoc Disord 2002; 16(2):103-108.

4. Nitrini R, Caramelli P, Herrera Jr E, Bahia VS, Caixeta LF, Radanovic M, Anghinah R, Charchat-Fichman H, Porto CS, Carthery MT, Hartmann AP, Huang N, Smid J, Lima EP, Takada LT, Takahashi DY. Incidence of Dementia in a Community-Dwelling Brazilian Population. Alzheimer Dis Assoc Disord 2004; 18(4):241-246.

5. Chaves ML, Camozzato AL, Godinho C, Piazenski I, Kaye J. Incidence of Mild Cognitive Impairment and Alzheimer Disease in Southern Brazil. J Geriatr Psychiatry Neurol 2009; 22(3):181-187.

6. Brasil. Ministério da Saúde. Portaria no 1.298, de 21 de novembro de 2013. Aprova o Protocolo Clínico e Diretrizes Terapêuticas da Doença de Alzheimer. Diário Oficial da União 2013; 24 out.

7. Ballard C, Gauthier S, Corbett A, Brayne C, Aarsland D, Jones E. Alzheimer's disease. Lancet 2011; 377(9770):1019-1031.

8. Sorbi S, Hort J, Erkinjuntti T, Fladby T, Gainotti G, Gurvit H. EFNS Scientist Panel on Dementia and Cognitive Neurology, et al. EFNS-ENS Guidelines on the diagnosis and management of disorders associated with dementia. Eur J Neurol 2012; 19(9):1159-1179.

9. Castro DM, Dillon C, Machnicki G, Allegri RF. The economic cost of Alzheimer's disease: Family or public-health burden? Dement Neuropsychol 2010; 4(4):262-267.

10. Alzheimer's Disease International. World Alzheimer Report 2010. The Global Economic Impact of Dementia. [acessado 2015 jan 26]. Disponível em: http://www. alz.org/documents/national/World_Alzheimer_Report_2010.pdf

11. Brasil. Ministério da Saúde. Portaria no 702, de 12 de abril de 2002. Dispõe sobre a criação de mecanismos para a organização e implantação de Redes Estaduais de Assistência à Saúde do Idoso. Diário Oficial da União 2002; 16 abr.

12. Brasil. Ministério da Saúde. Portaria no 843 , de 31 de outubro de 2002. Aprova o Protocolo Clínico e Diretrizes Terapêuticas da Doença de Alzheimer. Diário Oficial da União 2002; 4 nov.

13. Nacional Institute for Health and Clinical Excellence (NICE). NICE technology appraisal guidance 217: Donepezil, galantamine, rivastigmine and memantine for the treatment of Alzheimer's disease (review of NICE technology appraisal guidance 111). Londres: NICE/ NHS; 2014.

14. Winblad B, Grossberg G, Frölich L, Farlow M, Zechner S, Nagel J, Lane R. IDEAL: a 6-month, double-blind, placebo-controlled study of the first skin patch for Alzheimer disease. Neurology 2007; 69(4):14-22. 
15. Birks J. Cholinesterase inhibitors for Alzheimer's disease. Cochrane Database Syst Rev 2006; (1):CD005593.

16. Brasil. Ministério da Saúde. Portaria no 2.531, de 12 de Novembro de 2014. Redefine as diretrizes e os critérios para a definição da lista de produtos estratégicos para o Sistema Único de Saúde (SUS) e o estabelecimento das Parcerias para o Desenvolvimento Produtivo (PDP). Diário Oficial da União 2014; 13 nov.

17. Instituto de Pesquisa Econômica Aplicada (IPEA). Programas de assistência farmacêutica do Governo Federal: estrutura atual, evolução dos gastos com medicamentos e primeiras evidências de sua eficiência, 2005-2008. Brasília: IPEA; 2011.

18. Brasil. Lei $n^{\circ} 10.742$, de 6 de Outubro de 2003. Define normas de regulação para o setor farmacêutico, cria a Câmara de Regulação do Mercado de Medicamentos e altera a Lei $\mathrm{n}^{\circ} 6.360$, de 23 de setembro de 1976, e dá outras providências. Diário Oficial da União 2003; 7 out.

19. Brasil. Ministério da Saúde. Portaria no 2.981 de 26 de novembro de 2009. Aprova o Componente especializado da Assistência Farmacêutica. Brasília, Diário Oficial da União 2009; 1 dez.

20. Brasil. Ministério da Saúde. Portaria no 1.103, de 28 de maio de 2012. Estabelece processo de aquisição centralizada MS do medicamento rivastigmina $1,5 \mathrm{mg}, 3 \mathrm{mg}$, 4,5mg e 6 mg cáp, do CEAF. Diário Oficial da União 2012; 29 maio.

21. Brasil. Ministério da Saúde. Portaria no 1.554, de 30 de julho de 2013. Dispõe sobre as regras de financiamento e execução do Componente Especializado da Assistência Farmacêutica no âmbito do Sistema Único de Saúde. Diário Oficial da União 2013; 31 jul.

22. Brasil. Ministério da Saúde. Extrato de Convênio 00011/ 2012. Diário Oficial da União 2012; 16 ago.

23. Brasil. Ministério da Saúde. Extrato de Termo Aditivo 0001/2013 do Convênio 00011/2012. Diário Oficial da União 2013; 4 abr.

24. Araujo ALA, Pereira LRL, Ueta JM, Freitas O. Perfil da assistência farmacêutica na atenção primária do Sistema Único de Saúde. Cien Saude Colet 2008; 13(Supl.):611-617.

25. Vieira FS, Mendes ACR. Evolução dos gastos do Ministério da Saúde com medicamentos. Brasília: Ministério da Saúde; 2007.

26. Carias CM, Vieira FS, Giordano CV, Zucchi P. Medicamentos de dispensação excepcional: histórico e gastos do Ministério da Saúde do Brasil. Rev Saude Publica 2011; 45(2):233-240.
27. Brasil. Ministério da Saúde (MS). Da excepcionalidade às linhas de cuidado: o Componente Especializado da Assistência Farmacêutica. Brasília: MS; 2010.

28. Picon PD, Camozzato AL, Lapporte EA, Picon RV, Moser Filho H, Cerveira MO, Chaves ML. Increasing rational use of cholinesterase inhibitors for Alzheimer's disease in Brazil: public health strategy combining guideline with peer-review of prescriptions. Int $J$ Technol Assess Health Care 2010; 26(2):205-210.

29. Schneider LS, Dagerman KS, Higgins JT, McShane R. Lack of Evidence for the Efficacy of Memantine in Mild Alzheimer Disease. Arch Neurol 2011; 68(8):991-998.

30. Pepe VLE, Ventura M, Sant'ana JMB, Figueiredo TA, Souza VR, Simas L, Osório-de-Castro CGS. Caracterização de demandas judiciais de fornecimento de medicamentos "essenciais" no Estado do Rio de Janeiro, Brasil. Cad Saude Publica 2012; 26(3):461-471.

31. Pepe VLE, Figueiredo TA, Simas L, Osorio-de-Castro CGS, Ventura M. A judicialização da saúde e os novos desafios da gestão da assistência farmacêutica. Cien Saude Colet 2010; 15(5):2405-2414.

32. Brasil. Ministério da Saúde (MS). Componente Especializado da Assistência Farmacêutica: Inovação para a garantia do acesso a medicamentos no SUS. Brasília: MS; 2014.

33. Brasil. Ministério da Saúde. Portaria no 343, de 22 de fevereiro de 2010. Altera os Arts. $3^{\circ}, 15,16$ e 63 e o anexo IV à Portaria no 2.981 de 26 de novembro de 2009. Diário Oficial da União 2010; 24 fev.

34. Brasil. Ministério da Saúde. Portaria no 3.439 de 11 de novembro de 2010. Altera os Arts 30, 15, 16 e 63 e os Anexos I, II, III, IV e V à Portaria no 2.981/GM/MS, de 26 de novembro de 2009, republicada em $1^{\circ}$ de dezembro de 2009. Diário Oficial da União 2010; 11 nov.

Artigo apresentado em 01/04/2015

Aprovado em 05/08/2015

Versão final apresentada em 07/08/2015 